\title{
Camera calibration for miniature, low-cost, wide-angle imaging systems *
}

\author{
Oliver Frank, Roman Katz, Christel-Loic Tisse and Hugh Durrant-Whyte \\ ARC Centre of Excellence for Autonomous Systems \\ University of Sydney, Australia \\ \{o.frank, r.katz, c.tisse, h.durrant-whyte\}@cas.edu .au
}

\begin{abstract}
This paper presents a new model and an extension to an existing algorithm for camera calibration. The main goal of the proposed approach is to calibrate miniature, low-cost, wide-angle fisheye lenses. The model has been verified with a calibration implementation and was tested on real data. Experiments show that the proposed model improves the accuracy compared to the original algorithm. Results show that the extension not only performs well with fisheye lenses but also with omnidirectional catadioptric lenses as well as other less distorted dioptric lenses.
\end{abstract}

\section{Motivation and Related Work}

Wide-angle, hemispherical or omnidirectional camera systems have become more popular in the last few years. Especially in robotic applications, wide-angle sensors are favourable for perception and navigation problems. A precise calibration is needed in order to infer accurate bearing information for the $2 \mathrm{D}$ pixel information.

Three different types of calibration methods can be distinguished. The most common approaches are based on the correspondence of feature points whose world 3D coordinates are known $[14,10,4,6]$. These methods find the best external and internal parameters that correspond to the position of the feature points in the image. A second group of methods $[1,13]$ uses geometric invariants instead of world coordinates of image features (plumb lines, image of a sphere). The third group, the auto-calibration methods, does not need any kind of known feature points nor geometric invariants. Instead they only use constraints on the internal and external parameters. Their problem can be stability, as ambiguous motion sequences can lead to calibration degeneracies [3, 11].

Hemispherical or omnidirectional cameras imply additional challenges to the calibration procedure that go beyond affine or perspective projection. The single viewpoint constraint does not necessarily hold anymore and lens distortion plays a crucial role. To overcome the multiple viewpoint problem, non-central camera models were introduced. They project scenes onto images along a general set of rays that do not meet in a single point [7], [12]. An intermediate class of cameras are the axial cameras in which the projection rays intersect on a line [9].

\footnotetext{
*This work is supported by the ARC Centre of Excellence programme, funded by the Australian Research Council (ARC) and the New South Wales state government.
} 
The problem of image distortion caused by lens abberation or misalignment of the lenses has been studied extensively. Not only is the radial distortion of importance but also the decentering (aka tangential) distortion. The plumb lines were introduced in [2] which investigates the relation between straight lines in 3D and their distorted curved projection on the image sensor caused by lens distortion. Most of these models assume the centre of distortion to be at the principal point.

The proposed calibration tool presents an extension to the algorithm presented in [10] and is also motivated by the work of Bouguet ${ }^{1}$. It relies on the use of a planar calibration pattern. The only user input required is the selection of feature points with known 3D coordinates. More recently, an approach based on exact theoretical projection functions has been published in [6]. In this approach, deviations from the exact projection functions are modelled with added error parameters.

This paper is organised as follows. Section 2 introduces the proposed camera model. The process of estimating the calibration parameters is briefly explained in 3 and can be found in more detail in [10]. Section 4 shows experimental results with real data and compares the proposed calibration procedure with the original approach as well as with the algorithm presented in [6]. Finally, conclusions are drawn in Section 5.

\section{Camera Model}

The proposed model is based on the following assumptions: (i) the lens system has a single effective viewpoint, (ii) there is a misalignment of the image sensor with respect to the optical axis, (iii) non-radial distortion is ignored and the lens system is rotationally symmetrical with respect to its axis.

The first assumption of a single effective viewpoint is used for simplicity. Especially for miniature fisheye lenses with very short focal length this is a reasonable approximation. The second assumption is particularly motivated by low cost imaging systems. Misalignment can be caused by the inaccurate mounting of the lens as well as by misplacement of the image sensor relative to the optical axis. Contrary to the model presented in [10], the misalignment is modelled based on a perspective transformation instead of an affinity. Four degrees of freedom (DOF) are sufficient, as thoroughly explained in Section 2.3. The last assumption can be justified by the accurate manufacturing of lenses and the observation that non-radial distortion is mainly caused by the misalignment of different lens components.

The proposed camera model consists of the centre of distortion, the lens distortion function and the internal position of the sensor with respect to the lens. It is a good compromise between fully general camera models and conventional models in terms of complexity and stability of the calibration process.

\subsection{Geometry}

The proposed model can be described in two stages considering two different coordinate systems. The first one being the lens coordinate system $\left(X_{L}, Y_{L}, Z_{L}\right)$ which relates the orientation and position of the lens with respect to the world coordinates of the scene points

\footnotetext{
${ }^{1}$ Jean-Yves Bouguet. Camera Calibration Toolbox for Matlab, 2007 http://www.vision. caltech.edu/bouguetj/calib_doc/index.html
} 


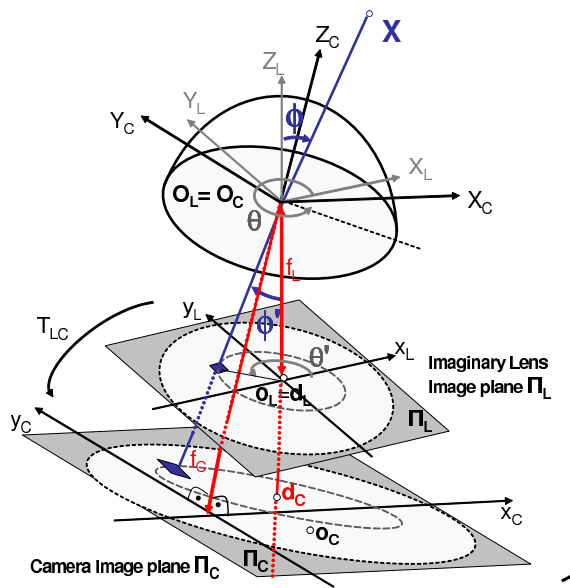

(a)

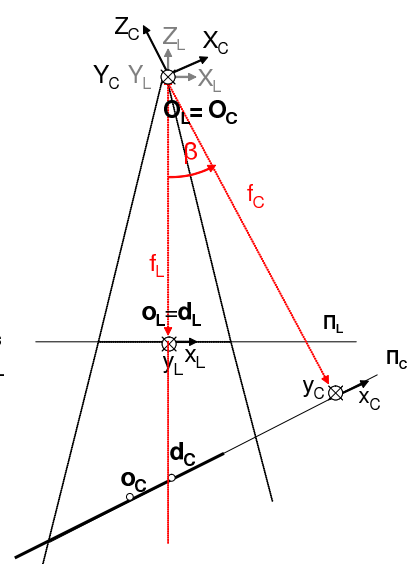

(b)

Figure 1: (a) Imaging model consisting of a radially symmetric distortion lens, an imaginary, perfectly aligned (principal point on optical axis and image plane perpendicular to the optical axis) lens image plane $\Pi_{L}$ and the misaligned physical image plane $\Pi_{C}$. (b) Top view for an example misalignment with rotation about $Y_{L}$ and change of focal length.

$\mathbf{X}$. The second coordinate system $\left(X_{C}, Y_{C}, Z_{C}\right)$ represents the orientation and position of the camera image sensor with respect to the lens coordinate system.

As illustrated in Figure 1, the incident 3D rays from scene points enter the lens system and, after a radial distortion, they are projected onto the imaginary lens image plane $\Pi_{L}$. $\Pi_{L}$ is the perpendicular plane to the optical axis $Z_{L}$, and the principal point $\mathbf{o}_{\mathbf{L}}$ is the intersection point of the optical axis $Z_{L}$ and the image plane $\Pi_{L}$. The distorted rays originating from the centre of projection $\mathbf{O}_{\mathbf{C}}$ do not physically intersect with the lens image plane $\Pi_{L}$ but with the camera image plane $\Pi_{C}$ instead. The physically captured image $\mathbf{x}_{\mathbf{C}}$ can be thought of a $3 \mathrm{D}$ perspective projection of the imaginary lens image $\mathbf{x}_{\mathbf{L}}$ with centre of projection $\mathbf{O}_{\mathbf{C}}$ equal to $\mathbf{O}_{\mathbf{L}}$. Therefore, the $3 \mathrm{D}$ transformation $T_{L C}$ from $\Pi_{L}$ to $\Pi_{C}$ can be described with a rotation and scale factor. Thus, only four parameters are sufficient to model the misalignment. In summary, the system can be described as: (1) Perfectly aligned projection with radially symmetric distortion, (2) Perspective projection of an imaginary planar scene $\Pi_{L}$ onto the camera image plane $\Pi_{C}$ with fixed centre of projection.

For fisheye lenses a perspective projection is more appropriate, contrary to other approaches, including the original algorithm presented in [10], where an affine projection is used to model misalignment. As explained in [5], the error between the true perspective image point and its affine approximation is given by

$$
\mathbf{x}_{\text {aff }}-\mathbf{x}_{\text {proj }}=\frac{\Delta}{d_{0}}\left(\mathbf{x}_{\text {proj }}-\mathbf{x}_{\mathbf{0}}\right),
$$

with $\Delta$ denoting the depth relief, $d_{0}$ the average depth and $\mathbf{x}_{\text {proj }}-\mathbf{x}_{\mathbf{0}}$ the distance of the point from the principal ray. For a misalignment between lens and sensor, the error is therefore inversely proportional to the focal length. Figure 2 shows the error of the affine 


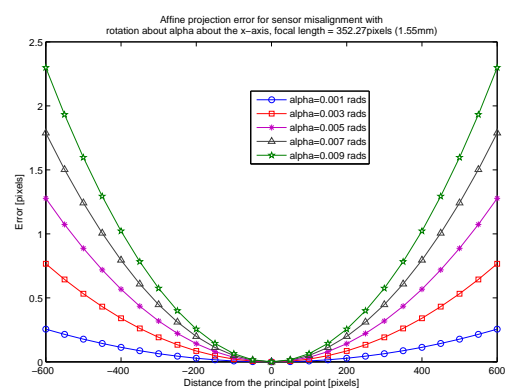

(a)

Figure 2: Error of affine projection versus distance from principal point. The errors are plotted for different misalignments due to a rotation $\alpha$ about the lens-axis $Y_{L}$.

projection for a variation of misalignments due to a rotation $\alpha$ about the lens-axis $Y_{L}$. The parameters were taken from a typical miniature, wide-angle imaging system as the one presented in Section 4. It can be seen that even for very small misalignments, the projection error is not negligible anymore.

\subsection{Perfectly aligned radially symmetric distortion}

For the first step of the calibration it is assumed that the distortion caused by the wideangle lens is radially symmetric and that the lens image is perfectly aligned with the optical axis of the lens. Rather than representing distortion as an image displacement it is modelled as a varying focal length, depending on the distance to the distortion center.

Let the azimuth angle of an incident ray from an object in the scene be $\theta$ and the polar angle $\phi$. Let $\mathbf{X}$ denote a $3 \mathrm{D}$ ray of a scene point in world coordinates and $\mathbf{x}_{\mathbf{L}}$ the corresponding distorted ray. Without further assumptions, the back projection of $\mathbf{x}_{\mathbf{L}}$ to $\mathbf{X}$ holds the relationship $\mathbf{x}_{\mathbf{L}}=\lambda \mathbf{X}$ :

$$
\mathbf{x}_{\mathbf{L}}=\left[\begin{array}{c}
x_{L} \\
y_{L} \\
g(\rho)
\end{array}\right] \simeq\left[\begin{array}{c}
X \\
Y \\
Z
\end{array}\right]=\mathbf{X},
$$

where $g(\rho)$ denotes the radial distortion function and $\rho=\sqrt{x_{L}^{2}+y_{L}^{2}}$ is the Euclidean distance from the centre of distortion $\mathbf{o}_{\mathbf{L}}$. The two rays $\mathbf{x}_{\mathbf{L}}$ and $\mathbf{X}$ are generally not equal but have the same direction. Therefore

$$
\tan (\phi)=\frac{\sqrt{X^{2}+Y^{2}}}{Z}=\frac{\sqrt{x_{L}^{2}+y_{L}^{2}}}{g(\rho)}=\frac{\rho}{g(\rho)} .
$$

As in [10], the distortion function $g(\rho)$ can be defined as

$$
g(\rho)=s_{0}+s_{2} \rho^{2}+\ldots+s_{N} \rho^{N},
$$

which satisfies $\partial g /\left.\partial \rho\right|_{\rho=0}=0$. By using (3) and (4)

$$
s_{0}-\tan (\pi / 2-\phi) \rho+s_{2} \rho^{2}+\ldots+s_{N} \rho^{N}=0,
$$


and therefore $\rho$ is found as the root of the above polynomial.

Without loss of generality, the focal length $f_{L}$ from the lens image to the centre of projection $\mathbf{O}_{\mathbf{L}}$ can be fixed to any value without affecting the calibration. For the case of no distortion, the model has to be coherent with the conventional perspective projection with a pinhole camera. Hence, the relation $g(\rho)=f_{L}$ can be found and by using (4), the focal length results in $f_{L}=s_{0}$. With this particular focal length, the relation between the incident angle $\phi$ of the 3D ray and the angle of the distorted ray $\phi^{\prime}$ can be explained by using (3):

$$
\tan \left(\phi^{\prime}\right)=\frac{\rho}{s_{0}},
$$

where $\rho$ can be found as the root of (5) and therefore only depends on the incident angle $\phi$ of the 3D ray.

The distorted image points on the lens image plane can be found using $\theta^{\prime}=\theta-\pi$, and equates to $\left[x_{L}, y_{L}\right]^{T}=\left[\rho \cos \left(\theta^{\prime}\right), \rho \sin \left(\theta^{\prime}\right)\right]^{T}$.

\subsection{Perspective projection onto the camera image sensor}

The same physical distorted rays $\mathbf{x}_{\mathbf{L}}$ as in Section 2.2 are now being projected onto the camera image sensor and converted in pixel coordinates. This can be thought of a perspective projection from the distorted rays onto the camera image plane.

Without loss of generality the focal length $f_{L}$ of the imaginary lens image plane can be set to 1 and thus the distorted rays $\mathbf{x}_{\mathbf{L}}$ can be written in homogenous coordinates as $\mathbf{x}_{\mathbf{L} h}=\left[x_{L}, y_{L}, 1,1\right]^{T}$. After a perspective projection with the centre of projection being $O_{C}=O_{L}$, the image points $\mathbf{x}_{\mathbf{C}}$ are found in homogenous coordinates as

$$
\mathbf{x}_{\mathbf{C} h}=\left[\begin{array}{lll}
x_{C} & y_{C} & w_{C}
\end{array}\right]^{T}=K_{C}\left[R_{L C} \mid 0\right] \mathbf{x}_{\mathbf{L h}}=K_{C} R_{L C} \mathbf{x}_{\mathbf{L}},
$$

where $R_{L C}$ is the $3 \times 3$ rotation matrix from the lens coordinate system to the camera coordinate system. It includes rotations $\alpha_{C}, \beta_{C}$ and $\gamma_{C}$ about the axis $x_{C}, y_{C}$ and $z_{C}$ respectively. $K_{C}$ is the common camera calibration matrix including the focal lengths $f_{C x}$ and $f_{C y}$ in pixel coordinates, $o_{C x}$ and $o_{C y}$, which are the coordinates of the principal point, and $s_{C}$ the skew parameter. In Euclidean coordinates (without $s_{C}, o_{C x}$ and $o_{C y}$ ), the projection from the lens coordinate system to the camera coordinate system has four degrees of freedom. It is fully described by a rotation and a scale factor.

\section{Calibration Procedure}

The calibration procedure presents an extension to the toolbox presented in [10]. It relies on a planar calibration grid which is captured from different poses. The algorithm is based on the popular method introduced by [14].

In order to fully calibrate the camera with the previously introduced model, estimations of all the $8+N+6 M$ parameters have to be performed. $M$ denotes the number of used calibration images and $N$ is the degree of the highest order term in (4). As found in [10] for the original algorithm and verified for the proposed calibration procedure, best approximations are expected with $N=4$. For one image $i$, the parameters are the conventional intrinsic ones $s, o_{c x}, o_{c y}$, the lens distortion parameters $s_{0}, s_{2}, \ldots, s_{N}$, the 
misalignment parameters $\alpha_{C}, \beta_{C}, \gamma_{C}, f_{C x}, f_{C y}$ and the conventional extrinsic parameters $\alpha^{i}, \beta^{i}, \gamma^{i}, C_{x}^{i}, C_{y}^{i}, C_{z}^{i}$. Note that a conventional perspective model has $5+6 M$ parameters.

The calibration procedure can be outlined as follows:

\section{Calibration Procedure:}

Given $M$ calibration images, determine the $8+N+6 M$ intrinsic parameters.

1. Estimation of the centre of distortion $\mathbf{d}$ for the aligned lens model as the least-squares solution of all images $I_{i}$ for $i=1 \ldots M$.

2. Linear estimation of the extrinsic parameters for all the images from $i=1 \ldots M$ by assuming the aligned model and using the previously estimated centre of distortion $\mathbf{d}$.

3. Linear estimation of the intrinsic parameters with the aligned lens model by using the previously estimated parameters for all images.

4. Optimised refinement and estimation of the remaining parameters with the full misaligned model. Use the linear estimations from 2 and 3 as initialisation values for the optimisation process.

Step 2 and 3 of the calibration procedure are similar to the one presented in [10] and thoroughly explained therein.

\subsection{Find the centre of distortion}

Step 1, the choice of the distortion centre is crucial for a stable and accurate estimation. As introduced in [4], the problem of radial distortion is similar to the motion of points seen by a camera moving forward towards a scene. The same idea can be used, even if an additional misalignment between the lens and the camera sensor is considered. The ideal, undistorted image $\mathbf{x}_{\mathbf{u}}$ is introduced as the projection of $\mathbf{X}$ with a perfect pinhole camera $\mathbf{x}_{\mathbf{u}}=\mathbf{P X}$. It is related to its radially distorted version $\mathbf{x}_{\mathbf{L}}$ on the imaginary image plane by $\mathbf{x}_{\mathbf{L}}=\mathbf{d}_{\mathbf{L}}+\lambda(\phi) \cdot\left(\mathbf{x}_{\mathbf{u}}-\mathbf{d}_{\mathbf{L}}\right)$, with $\mathbf{d}_{\mathbf{L}}$ being the distortion centre, and $\lambda(\phi)=\rho / r$ the radially symmetric distortion factor from the distorted to the undistorted Euclidean radius. By writing the relation between the distorted image $\mathbf{x}_{\mathbf{L}}$ and its misaligned version $\mathbf{x}_{\mathbf{C}}$ in (7) as a homography $\mathbf{x}_{\mathbf{C}}=\mathbf{H}_{\mathbf{L C}} \mathbf{x}_{\mathbf{L}}$, the following relation can be found:

$$
\mathbf{H}_{\mathbf{L C}}^{-1} \mathbf{x}_{\mathbf{C}}=\mathbf{d}_{\mathbf{L}}+\lambda(\phi) \cdot\left(\mathbf{x}_{\mathbf{u}}-\mathbf{d}_{\mathbf{L}}\right)
$$

Multiplying this expression on the left by the skew-symmetric matrix $\left[\mathbf{d}_{\mathbf{L}}\right]_{x}$, and substituting $\mathbf{x}_{\mathbf{u}}$ by $\mathbf{P X}$ equates in

$$
\left[\mathbf{d}_{\mathbf{L}}\right]_{x} \mathbf{H}_{\mathbf{L C}}^{-\mathbf{1}} \mathbf{x}_{\mathbf{C}}=\lambda(\phi)\left[\mathbf{d}_{\mathbf{L}}\right]_{x} \mathbf{P X}
$$

Another multiplication on the left by $\left(\mathbf{H}_{\mathbf{L C}}^{-\mathbf{1}} \mathbf{x}_{\mathbf{C}}\right)^{T}$ results in

$$
0=\lambda(\phi) \mathbf{x}_{\mathbf{C}}^{T}\left(\mathbf{H}_{\mathbf{L C}}^{-\mathbf{T}}\left[\mathbf{d}_{\mathbf{L}}\right]_{x} \mathbf{P}\right) \mathbf{X}
$$

This can be compared to the usual fundamental matrix relation $\mathbf{x}_{\mathbf{L}}{ }^{T} \mathbf{F} \mathbf{x}_{\mathbf{C}}=0$ with $\mathbf{F}$ being the substitution of $\mathbf{H}_{\mathbf{L C}}^{-\mathbf{T}}\left[\mathbf{d}_{\mathbf{L}}\right]_{x} \mathbf{P}$. It can be computed from several point correspondences between the calibration pattern $\mathbf{X}$ and the distorted points $\mathbf{x}_{\mathbf{C}}$ in the camera image plane [5]. A final multiplication on the left by $\mathbf{H}_{\mathbf{L C}}^{\mathbf{T}} \mathbf{d}_{\mathbf{L}}{ }^{T}$ yields

$$
\left(\mathbf{H}_{\mathbf{L C}} \mathbf{d}_{\mathbf{L}}\right)^{T} \mathbf{F}=0
$$

and the centre of distortion in the misaligned camera image plane can be found as the left null space generator of $F$. This can be done with the singular value decomposition of $F$. 


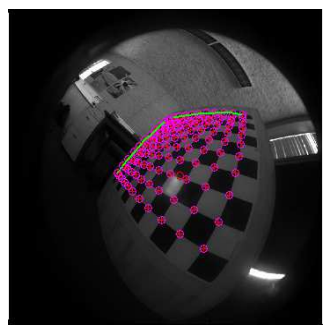

(a)

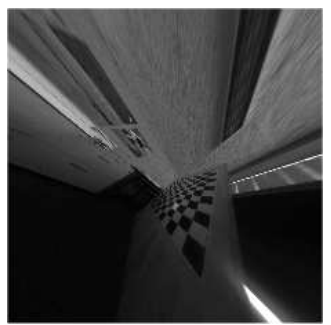

(b)

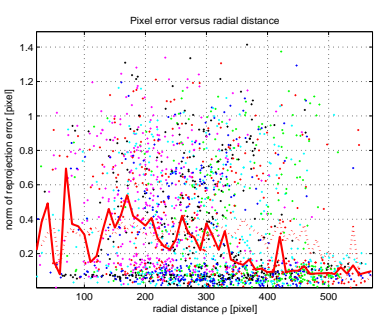

(c)

Figure 3: (a) Sample calibration image with extracted and reprojected grid points overlaid. (b) Image after rectification. (c) Radial distribution of the reprojection errors for all images contained in the set.

\subsection{Full misaligned model and non-linear refinement}

The final step of the calibration process refines the previously estimated intrinsic and extrinsic parameters that were found by assuming a perfectly aligned system. Simultaneously the remaining intrinsic parameters $f_{C x}, f_{C x}, \alpha_{C}, \beta_{C}$ and $\gamma_{C}$ for the full misaligned camera model are now estimated. This can be done either for the intrinsic and extrinsic parameters independently, or simultaneously with bundle adjustment. The latter is expected to provide more accurate results at higher computational cost. For the experimental results in Section 4 the Levenberg-Marquardt [8] method was used to refine all parameters simultaneously.

\section{Experimental Results}

The proposed camera model and the calibration algorithm were tested on real images from a planar calibration checkerboard. A simple corner detector was used to extract the 'ground truth' grid corners. These values were then compared with the calculated reprojections from the known world coordinates to the camera pixel coordinates. As a performance measurement the mean square reprojection error in pixel coordinates was used. In the following figures the 'ground truth' and reprojected grid corners are represented as ('+') and ('o') respectively.

The first and second data sets, Fisheye 1 and Fisheye2, were taken with a uEye UI2250 from Imaging Development Systems together with a miniature fisheye lens DSL215 from Sunex. The field of view for the miniature fisheye lens is specified at about $185^{\circ}$ and the focal length is $1.55 \mathrm{~mm}$. The entire data set contains 18 images. Figure 3(a) shows a sample image taken from the imaging system with the extracted corner points (+) and its reprojection (o). Figure 3(b) illustrates the calculated rectified image as if viewed with a perfect pinhole camera system. The radial distribution of the reprojection error for all calibration images in this set is depicted in Figure 3(c). The solid line represents the median value. The undistorted incident angle $\phi$ versus the distorted angle $\phi^{\prime}$ is plotted in Figure 5 (a). It can be seen that the field of view is about $186.8^{\circ}$ compared to the specified $185^{\circ}$.

The data set Subset1 is a subset of Fisheyel with only four images. This data set was 


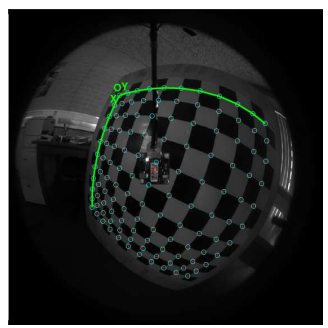

(a)

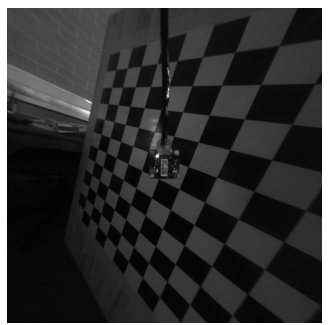

(b)

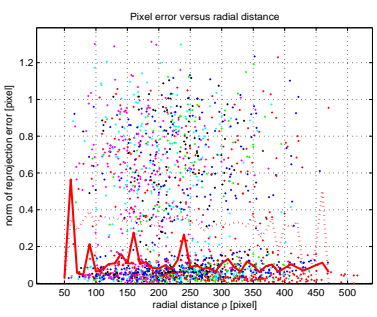

(c)

Figure 4: (a) Sample calibration image with extracted and reprojected grid points overlaid. (b) Image after rectification. (c) Radial distribution of the reprojection errors for all images contained in the set.

\begin{tabular}{|c|c|c|c|}
\hline & Scaramuzza [10] & Mei [6] & Proposed \\
\hline Fisheye1 & $0.434 \pm 0.290$ & n/a & $0.352 \pm 0.310$ \\
Subset1 & $0.354 \pm 0.288$ & $0.334 \pm 0.293$ & $0.272 \pm 0.310$ \\
Fisheye2 & $0.412 \pm 0.298$ & n/a & $0.301 \pm 0.324$ \\
Parabolic & $0.343 \pm 0.201$ & $0.291 \pm 0.227$ & $0.319 \pm 0.300$ \\
Digiclops & $0.153 \pm 0.101$ & n/a & $0.148 \pm 0.102$ \\
\hline
\end{tabular}

Table 1: Comparison of the mean squared reprojection error and its standard deviation in pixels for all data sets.

produced in order to allow a comparison with the algorithm presented in [6] as further explained below.

The second data set, Fisheye2, was taken with a second imaging system similar to the first one. In this setup, partial occlusion was an additional challenge as not all grid points are visible in the centre area of the image. Figure 4 shows one example image from the data set containing 18 images. The sample image with the reprojected grid points overlaid is shown in Figure 4(a). Figure 4(b) represents the rectified image and (c) depicts the radial distribution of the reprojection error for the whole set.

The third data set ${ }^{2}$, Parabolic, was captured with a parabolic mirror with a diameter of $80 \mathrm{~mm}$ and a focal distance of $16.7 \mathrm{~mm}$. The set contains 10 images.

The fourth data $\operatorname{set}^{3}$, Digiclops, contains images taken with a Digiclops camera from Point Grey Research. This imaging system presents only very small distortion effects. The radial distribution of the mean squared reprojection error is illustrated in Figure 5 (b) for the third and in (c) for the fourth data set.

The proposed calibration procedure was compared to the algorithm in [10] and in [6]. Both implementations are online available as a Matlab toolbox. The toolbox from [6] does not provide an estimation for the centre of distortion and the initialisation was done manually with the results from the proposed calibration procedure. Further, the toolbox does not allow to extract grid points manually. Only the grid points of four of the images

\footnotetext{
${ }^{2}$ Dataset kindly provided by C. Mei, http://www.robots.ox.ac.uk/ cmei/Toolbox.html

${ }^{3}$ Dataset kindly provided from ACFR, The University of Sydney and LCR, Universidad Nacional del Sur. PAATV/UTE Projects, 2006.
} 


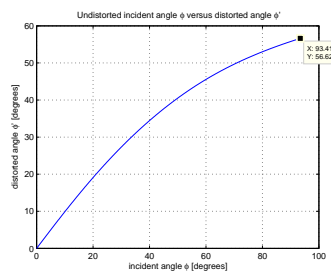

(a)

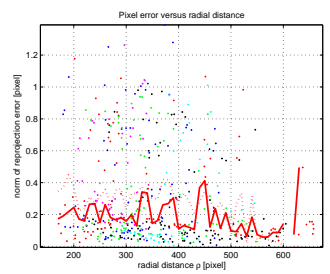

(b)

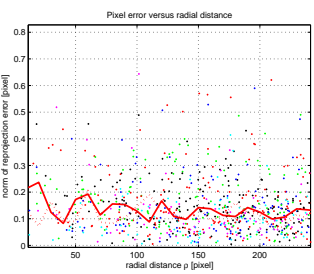

(c)

Figure 5: (a) Incident angle $\phi$ versus distorted angle $\phi^{\prime}$ for the first data set. Radial distribution of the reprojection error for the Parabolic mirror data set in (b) and for the Digiclops data set in (c).

in Fisheyel could be extracted successfully. To allow a comparison nevertheless, Subset 1 was created. Because of missing extrinsic parameters for the remaining images, their grid points could not be extracted. The data set Fisheye 2 could not be applied because of partial occluded grid points. The iterative procedure to find the centre of distortion in the toolbox from [10] failed for the data sets Parabolic as well as Fisheye2. They were initialised with the results from the other algorithms. For Fisheye2, the results from the proposed calibration procedure were used and for Parabolic the results from the toolbox from [6] were used.

Table 1 summarises the mean-squared reprojection errors and its standard deviation for all data sets. It can be seen that the proposed calibration procedure performs well with the miniature, low-cost, wide-angle imaging systems as in data set Fisheyel and Fisheye2. Please note that the results from Subset1 might not be representative as the number of calibration images is not sufficient. The data sets Parabolic and Digiclops were used to show that the new model also provides good results for less complex systems (less misalignment, less distortion, and increased focal length) where the additional parameters might be redundant.

\section{Summary and Conclusion}

An extension to an existing calibration algorithm has been proposed in order to deal with miniature, low-cost, and wide-angle imaging systems. Experimental results with different data sets and a comparison with other algorithms showed that the proposed procedure improves the accuracy for the targeted imaging systems. The proposed camera model also performs well for less complex systems as omnidirectional mirror systems or less distorted cameras.

\section{References}

[1] J.P. Barreto and H. Araujo. Paracatadioptric camera calibration using lines. IEEE International Conference on Computer Vision, 2, 2003.

[2] D.C. Brown. Lens distortion for close-range photogrammetry. Photometric Engineering, 37(8):855-866, 1971. 
[3] O.D. Faugeras, Q. Luong, and S.J. Maybank. Camera self-calibration: Theory and experiments. In European Conference on Computer Vision, pages 321-334, London, UK, 1992. Springer-Verlag.

[4] R. Hartley and S. B. Kang. Parameter-free radial distortion correction with centre of distortion estimation. Technical Report MSR-TR-2005-42, Microsoft Research, April 2005.

[5] R. Hartley and A. Zisserman. Multiple View Geometry in Computer Vision. Cambridge University Press, 2nd edition, March 2004.

[6] C. Mei and P. Rives. Single view point omnidirectional camera calibration from planar grids. In IEEE International Conference on Robotics and Automation, April 2007. To appear.

[7] B. Micusik and T. Pajdla. Autocalibration \& 3d reconstruction with non-central catadioptric cameras. In IEEE International Conference on Computer Vision and Pattern Recognition, volume 1, pages 58-65, Los Alamitos, CA, USA, 2004. IEEE Computer Society.

[8] J. Nocedal and S.J. Wright. Numerical Optimization. Springer Series in Operations Research. Springer-Verlag, 1999.

[9] S. Ramalingam, P. Sturm, and S. Lodha. Theory and calibration for axial cameras. In Proceedings of the Asian Conference on Computer Vision, Hyderabad, India, volume 1, pages 704-713, January 2006.

[10] D. Scaramuzza, A. Martinelli, and R. Siegwart. A toolbox for easily calibrating omnidirectional cameras. In IEEE International Conference on Intelligent Robots and Systems, pages 5695-5701, 2006.

[11] G. P. Stein. Accurate internal camera calibration using rotation, with analysis of sources of error. In IEEE International Conference on Computer Vision, page 230, Washington, DC, USA, 1995. IEEE Computer Society.

[12] P. Sturm and S. Ramalingam. A generic concept for camera calibration. In Proceedings of the European Conference on Computer Vision, Prague, Czech Republic, volume 2, pages 1-13. Springer, May 2004.

[13] X. Ying and Z. Hu. Catadioptric camera calibration using geometric invariants. IEEE Transactions on Pattern Analysis and Machine Intelligence, 26(10):1260$1271,2004$.

[14] Z. Zhang. A flexible new technique for camera calibration. Technical Report MSRTR-98-71, Microsoft Research, December 1998. 